PROCEEDINGS OF THE

AMERICAN MATHEMATICAL SOCIETY

Volume 80, Number 1, September 1980

\title{
COMPACITÉ EXTRÊMALE
}

\author{
JEAN BOURGAIN ET MICHEL TALAGRAND
}

RÉSumé. Un ensemble borné d'un espace de Banach $E$ est faiblement compact dès qu'il est compact pour la topologie de la convergence simple sur les points extrêmaux de la boule unité du dual de $E$.

Soit $X$ un espace compact; munissons $\mathcal{C}(X)$ de la norme uniforme. La topologie de la convergence ponctuelle sur $X$ n'est autre que la topologie de la convergence sur les points extrêmaux de la boule unité du dual de $\mathcal{C}(X)$. Ainsi si $E$ est un espace de Banach, il est natural de considérer la topologie $e(E)=\sigma\left(E, \mathcal{E}_{E}\right)$, dite extrêmale, de la convergence simple sur l'ensemble $\mathcal{E}_{E}$ des points extrêmaux de la boule unité de $E^{\prime}$. (Cette topologie dépend donc de la norme de $E$.)

Si $E=C(X)$, un théorème classique de Grothendieck affirme que tout ensemble borné, compact pour la topologie de la convergence ponctuelle est faiblement compact. Il est donc naturel de poser le problème suivant: Pour tout espace de Banach, un ensemble borné et $e(E)$ compact est-il faiblement compact? (L'idée de considérer la topologie extrêmale ainsi que ce problème, sont dû à $\mathbf{H}$. Kharaghani.)

ThÉorème 1. Soit $E$ un espace de Banach, et $K$ un ensemble borné et e(E) dénombrablement relativement compact de $E$. Alors $E$ est faiblement relativement compact.

Preuve. La difficulté est que les sous-espaces fermés de $E$ ne sont pas nécessairement $e(E)$-fermés.

Remarquons d'abord que si $F \subset E, e(F)$ est moins fine que la restriction de $e(E)$ à $F$. En effet pour tout point extrémal $t$ de la boule unité de $F^{\prime}$, l'ensemble des points de la boule unité de $E^{\prime}$ dont la restriction à $F$ est égale à $t$, est une face de cette boule unité, donc contient un point extrémal. (En général $e(F)$ est strictement moins fine que la restriction de $e(E)$.)

Soit $x_{n}$ une suite de $K$. Il suffit d'après le théorème d'Eberlein, de montrer qu'elle a une sous-suite faiblement convergente. Désignons par $F$ l'espace engendré par les $x_{n}$. La boule unité $B_{F}^{\prime}$ de $F^{\prime}$, munie de la topologie préfaible $\sigma\left(F^{\prime}, F\right)$, est un convexe compact métrisable. L'ensemble $X$ de ses points extrêmaux est donc un $G_{\delta}$, et donc un espace Polonais.

Supposons d'abord, qu'aucune sous-suite de la suite $x_{n}$ ne converge sur $X$. D'après le théorème 4D de [2], il existe un compact $L$ de $X$ tel que l'ensemble $y_{n}$ des restrictions de $x_{n}$ à $L$ ne possède pour la topologie de la convergence simple, aucune valeur d'adhérence borélienne.

Received by the editors May 16, 1979 and, in revised form, September 19, 1979.

AMS (MOS) subject classifications (1970). Primary 46A50, 46A55.

Key words and phrases. Extreme point, integral representation.

(C) 1980 American Mathematical Society 0002-9939/80/0000-0411/\$01.75 
Soit $z$ un point $e(E)$ adhérent à la suite $\left(x_{n}\right)$ dans $E$. Soit $G$ l'espace engendré par $z$ et les $\left(x_{n}\right)$. Alors $z$ est $e(G)$ adhérent aux $\left(x_{n}\right)$. Soit $Y$ l'ensemble des points extrêmaux de la boule unité de $G^{\prime}$, et $p$ l'application restriction de $G^{\prime}$ à $F^{\prime}$. Comme nous l'avons $v u, X \subset p(Y)$. Soit $M=p^{-1}(L)$. C'est un espace Polonais. La restriction $u$ de $z$ à $M$ est par hypothèse adhérente (pour la convergence simple) aux fonctions $y_{n} \circ p$ sur $M$. Puisque $p(M)=L$, on peut écrire $u=v \circ p$, où $v$ est adhérente aux $y_{n}$. Pour tout borelien $H$ de $\mathbf{R}, v^{-1}(H)=p\left(u^{-1}(H)\right)$ est un ensemble analytique. Puisque il en est de même de $v^{-1}(\mathbf{R} \backslash H)$, le théorème de séparation des ensembles analytiques [3] montre que $v$ est borelienne sur $L$, ce qui est impossible.

Nous avons ainsi montré que $\left(x_{n}\right)$ possède une sous-suite, que l'on peut encore appeler $\left(x_{n}\right)$, qui converge simplement sur $X$. D'après le théorème de représentation intégrale de Choquet [1] tout élément de la boule unité de $F^{\prime}$ est barycentre d'une mesure sur $X$. Le théorème de Lebesgue montre alors que la suite $\left(x_{n}\right)$ est de Cauchy faible dans $F$ donc dans $E$. Ainsi elle admet une valeur d'adhérence unique $z$ pour $e(E)$. Désignons par $G$ l'espace engendré par $z$ et les $\left(x_{n}\right)$.

Alors $x_{n}$ converge vers $z$ pour $e(G)$ (qui est moins fine que $e(E)$ ) et un nouvel appel au théorème de représentation intégrale montre que $x_{n}$ converge faiblement vers $z$ dans $G$, donc dans $E$. Q.E.D.

Voici une conséquence intéressante de ce résultat.

ThÉorème 2. Soit $K$ un espace compact et $A$ un convexe uniformément bornè de fonctions réelles sur $K$. Supposons que A soit compact pour la topologie $\tau_{p}$ de la convergence ponctuelle sur $K$. Alors si tout point extrêmal de $K$ est une fonction continue, $A$ est formé de fonctions continues.

Preuve. Tout point extrémal de l'enveloppe convexe équilibrée de $A$ est un point extrémal de $A$ ou $-A$. On peut donc supposer $A$ équilibré. Munissons-le de la topologie $\tau_{p}$, et désignons par $E$ l'espace de Banach des fonctions affines continues sur $A$ qui sont nulles en zéro. Il est aisé de voir que $A$ s'identifie à la boule unité de $E^{\prime}$ munie de la topologie préfaible. L'application $\varphi$ de $K$ dans $E$, qui envoie $t$ sur la restriction de la mesure de Dirac en $t$ à $A$, est continue lorsque $E$ est muni de $e(E)$, et ainsi $\varphi(K)$ est $e(E)$-compacte. De plus $\varphi(K)$ est bornée, et le Théorème 1 montre alors que $\varphi(K)$ est faiblement compacte, et que $e(E)$ et la topologie faible coincident sur $\varphi(K)$. Ainsi $\varphi$ est continue lorsque $K$ est muni de la topologie faible, c'est à dire que $A \subset \mathcal{C}(K)$.

Le résultat suivant généralise le Théorème (3-4) de [4].

ThÉorèmE 3. Soit $E$ un espace de Banach. Alors $E$ est faiblement $\mathcal{H}$-analytique dès que $E$ est $e(E)-\mathcal{H}$-analytique.

Preuve. La boule unité $B$ de $E$ est $e(E)$-fermée. Elle est donc $e(E)$ - $\mathcal{H}$-analytique. Il résulte du Théorème (2-1) de [4] et du Théorème 1 qu'elle est faiblement $\mathcal{H}$-analytique, ce qui suffit. 


\section{REFERENCES}

1. G. Choquet, Lectures on analysis, Benjamin, New York, 1969.

2. J. Bourgain, D. H. Fremlin and M. Talagrand, Pointwise compact sets of Baire measurable functions, Amer. J. Math. 100 (1978), pp. 845-886.

3. K. Kuratovski, Topology, Vol. I, Academic Press, New York, 1966.

4. M. Talagrand, Espaces de Banach faiblement $\mathcal{X}$-analytiques, Ann. of Math. (a paraitre)

Departement de Mathematiques, Vrife Universiteit Brussel, Plennlann 2-F7, 1050 Bruxalles, BELGIUM

Equipe d'Analyse, Université Paris 6, 75230 Paris, Cédex 05, France 